\title{
System Level Design of a Continuous-Time Delta-Sigma Modulator for Portable Ultrasound Scanners
}

Llimos Muntal, Pere; Færch, Kjartan; Jørgensen, Ivan Harald Holger; Bruun, Erik

Published in:

Proceedings of NORCAS 2015

Link to article, DOI:

10.1109/NORCHIP.2015.7364408

Publication date:

2015

Document Version

Peer reviewed version

Link back to DTU Orbit

Citation (APA):

Llimos Muntal, P., Færch, K., Jørgensen, I. H. H., \& Bruun, E. (2015). System Level Design of a ContinuousTime Delta-Sigma Modulator for Portable Ultrasound Scanners. In Proceedings of NORCAS 2015 IEEE. https://doi.org/10.1109/NORCHIP.2015.7364408

\section{General rights}

Copyright and moral rights for the publications made accessible in the public portal are retained by the authors and/or other copyright owners and it is a condition of accessing publications that users recognise and abide by the legal requirements associated with these rights.

- Users may download and print one copy of any publication from the public portal for the purpose of private study or research.

- You may not further distribute the material or use it for any profit-making activity or commercial gain

- You may freely distribute the URL identifying the publication in the public portal 


\title{
System Level Design of a Continuous-Time $\Delta \Sigma$ Modulator for Portable Ultrasound Scanners
}

\author{
Pere Llimós Muntal*, Kjartan Færch ${ }^{\dagger}$, Ivan H.H. Jørgensen* and Erik Bruun* \\ * Department of Electrical Engineering, Technical University of Denmark, Kgs. Lyngby, Denmark \\ $\dagger$ Analogic Ultrasound, BK Medical Design Center, Herlev, Denmark \\ plmu@elektro.dtu.dk, kfaerch@bkultrasound.com, ihhj@elektro.dtu.dk, eb@elektro.dtu.dk
}

\begin{abstract}
In this paper the system level design of a continuous-time $\Delta \Sigma$ modulator for portable ultrasound scanners is presented. The overall required signal-to-noise ratio (SNR) is derived to be $42 \mathrm{~dB}$ and the sampling frequency used is $320 \mathrm{MHz}$ for an oversampling ratio of 16. In order to match these requirements, a fourth order, 1-bit modulator with optimal zero placing is used. An analysis shows that the thermal noise from the resistors and operational transconductance amplifier is not a limiting factor due to the low required SNR, leading to an inherently very low-power implementation. Furthermore, based on high-level VerilogA simulations, the performance of the $\Delta \Sigma$ modulator versus various block performance parameters is presented as trade-off curves. Based on these results, the block specifications are derived.
\end{abstract}

\section{INTRODUCTION}

Ultrasound systems are widely used in medical applications as a diagnosis technique. It has many advantages such as non-invasing scanning, live imaging and no long-term effect on the patient. Furthermore, the scanning equipment to perform ultrasound imaging is easily accessible and inexpensive compared to other diagnosis techniques like $\mathrm{x}$-ray. However, ultrasound scanners are static devices with a significant size and high power consuming, which limits the amount of diagnosis that can be performed per unit of time. For the purpose of lowering the cost and increasing the amount of diagnosis per unit of time, portable ultrasound devices are being developed. Nonetheless, portable ultrasound scanners have a size limitation and are supplied with a battery which imposes another limitation on the maximum power consumption of the electronics inside. In order to maximize the quality of the picture with a fixed power budget, the electronics need to be custom designed, hence an application specific integrated circuit (ASIC) solution is required.

Ultrasound scanners consist of a transmitting circuit (Tx) [1], [2], a receiving circuit $(\mathrm{Rx})$ and a transducer. In transmitting mode the transducer gets excited by the high-voltage Tx generating ultrasonic waves. In receiving mode the lowvoltage $\mathrm{Rx}$ amplifies, delays and digitizes the waves received by the transducer. The Rx is usually the most power consuming circuitry due to the high receiving duty cycle of ultrasound scanners. One of the highest power consuming block of the receiving circuitry is typically the $\mathrm{ADC}$, hence it is a very critical design for portable ultrasound scanners.

This paper presents the design of a fully-differential continuous-time delta-sigma modulator (CTDSM) for a receiving channel of a portable ultrasound scanners using capacitive ultrasonic micromachined transducers (CMUTs).

\section{SYSTEM LEVEL ADC REQUIREMENTS}

The CTDSM in this paper is designed specifically for the 64-channel ultrasound Rx system in Fig. 1. Each channel contains a CMUT, a low noise amplifier (LNA), a time-gain control (TGC), an analog to digital converter (ADC) and a digital delay (DD). All channels are digitally summed using beamforming in order to reduce the amount of data that needs to be transferred from the portable device to the digital signal processing unit. The signal to noise ratio (SNR) of this data dictates the maximum image quality achievable, however, the higher the SNR the more power consuming the electronics are. The design target is to achieve the lowest power consumption with an acceptable level of image quality, which is estimated to be obtained with a minimum of $60 \mathrm{~dB}$ SNR at the output $\left(\mathrm{SNR}_{\text {out }}\right)$. Nonetheless, the signals received by the CMUT are uncorrelated, hence the SNR after summing $2^{\mathrm{N}}$ channels is $\mathrm{N} \cdot 3 \mathrm{~dB}$ higher than the single channel SNR. In this particular ultrasound receiving system, if a $\mathrm{SNR}_{\text {out }}$ of $60 \mathrm{~dB}$ wants to be achieved, SNR of each ADCs needs to be $42 \mathrm{~dB}$.

The supply rails of the electronics in the $\mathrm{Rx}$ system are specified at $\mathrm{V}_{\mathrm{ss}}=0 \mathrm{~V}$ and $\mathrm{V}_{\mathrm{dd}}=1.2 \mathrm{~V}$ with a common mode level of $\mathrm{V}_{\mathrm{cm}}=0.6 \mathrm{~V}$. The input signal of the fully-differential ADC, which is defined by the output signal of the TGC, is a differential signal with a $10 \mathrm{MHz}$ bandwidth (BW) and peakto-peak voltage of $\mathrm{V}_{\mathrm{pp}}=1.2 \mathrm{~V}$.

Another important specification of the Rx system is the delay resolution in the $\mathrm{DD}$, which determines the precision of the beamforming. Increasing the resolution of the delay improves the image resolution but it also increases the power consumption and area of the digital circuitry. A study performed showed that the minimum delay resolution that provides a sufficient image quality is $3 \mathrm{~ns}$. This result has a large impact on the ADC topology selection.

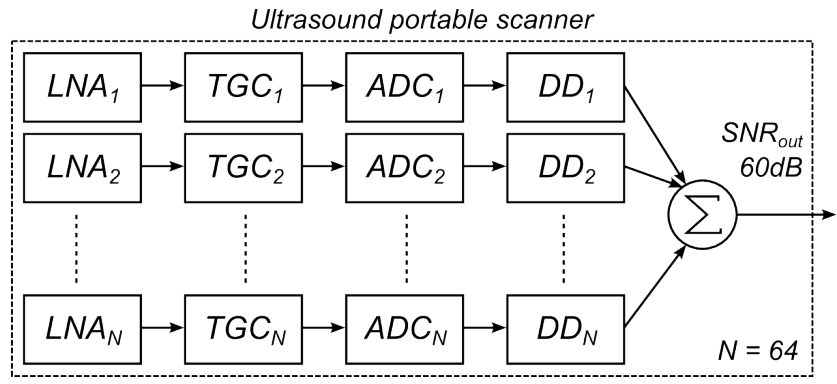

Fig. 1. 64-channel ultrasonic portable device structure. 
TABLE I. Continuous-Time $\Delta \Sigma$ Modulator SPECIFICATIONS

\begin{tabular}{|c|c|c|c|c|c|}
\hline SNR [dB] & BW [MHz] & $\mathbf{V}_{\mathbf{p p}}[\mathbf{V}]$ & $\mathbf{V}_{\mathbf{c m}}$ [V] & OSR & Quant. bits \\
\hline 42 & 10 & 1.2 & 0.6 & 16 & 1 \\
\hline
\end{tabular}

After determining the specifications of the ADC, a topology must be chosen. Traditionally a nyquist-rate ADC running at two times the BW $(20 \mathrm{MHz})$ is used. However, the delay resolution achievable is only $50 \mathrm{~ns}$ hence there is a need for an interpolation filter. These filters are complex, area demanding and power consuming. An alternative approach is to use a delta-sigma modulator with an oversampling ratio (OSR) of 16 running at a sampling frequency $\mathrm{f}_{\mathrm{s}}=320 \mathrm{MHz}$, which inherently provides enough delay resolution. A continuoustime delta-sigma modulator is selected over a discrete-time due to its lower power and higher frequency operation range [3], [4]. In order to simplify the digital circuitry the number of bits in the output of the delta-sigma modulator is chosen to be 1. In this case, the DD block becomes a simple 1-bit delay line running at $320 \mathrm{MHz}$ which can be easily be accessed at any intermediate point, and can be custom designed to achieve high efficiency. A summary of the specifications of the CTDSM is shown in Table I.

\section{Continuous-Time $\Delta \Sigma$ Modulator Design}

The first step of designing a CTDSM is to split total noise budget, $\mathrm{SNR}_{\mathrm{tot}}$, into quantization and thermal noise. Typically, the signal to quantization noise ratio (SQNR) is designed to be $10-12 \mathrm{~dB}$ higher than the target $\mathrm{SNR}_{\text {tot }}$, allowing for the thermal noise to spend most of the noise budget. This margin is used later in the implementation in order to accommodate for circuitry with non ideal specifications. In this design, for a total $\mathrm{SNR}_{\text {tot }}$ of $42 \mathrm{~dB}$, the SQNR targeted is $54 \mathrm{~dB}$, which leads to a maximum spectral density of the thermal noise of $3.3 \mathrm{mV} / \sqrt{\mathrm{Hz}}$.

The following step is to determine the order (M) and output of band gain of the loop filter $\left(\mathrm{H}_{\text {inf }}\right)$ of the CTDSM. For that purpose a discrete-time model of the CTDSM is used. In Fig. 2 the SQNR and the maximum stable amplitude (MSA) are plotted versus the $\mathrm{H}_{\text {inf }}$ for different orders. The OSR is set to 16 and number of output bits is set to 1-bit for all the plots. Optimal placing of zeros is used for all the orders to obtain a
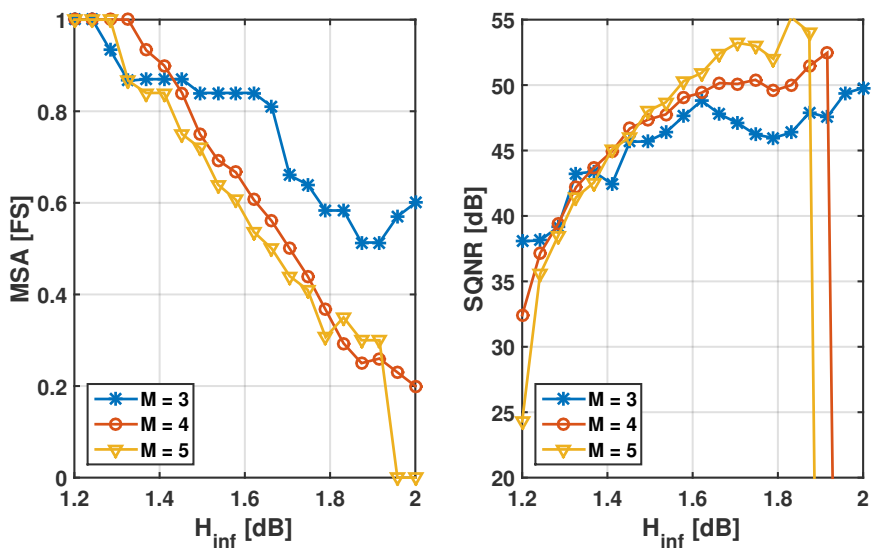

Fig. 2. MSA and SQNR at MSA-6 dB versus $H_{\text {inf }}$ for different $M$.

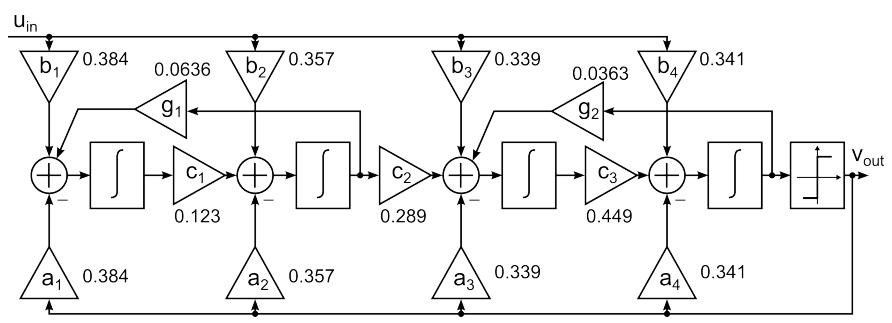

Fig. 3. Structure of the continuous-time delta-sigma modulator.

higher SQNR [3]. As it can be seen from Fig. 2 the minimum order that can achieve a sufficient peak SQNR is $M=4$, and $\mathrm{H}_{\mathrm{inf}}=1.7 \mathrm{~dB}$ leads to the best compromise between SQNR and MSA. A low MSA can be chosen due to the high thermal noise allowed in the circuitry.

The structure chosen to implement the CTDSM is the cascade-of-resonators feedback structure (CRFB) shown in Fig. 3. It consists of four feedforward paths, $a_{1}-a_{4}$, four feedback paths $b_{1}-b_{4}$, three scaling coefficients $c_{1}-c_{3}$ and two resonators $g_{1}-g_{2}$. Feedforward was used so that the integrators only have to process the noise and not the input signal, hence their output swing is reduced. The two resonator coefficients realize the optimal placing of the zeros of the system. The value of the continuous-time coefficients of this CRFB structure can also be seen in Fig. 3. Using this structure and coefficients, the frequency spectrum of the continuous time model of the CTDSM is shown in Fig. 4. The MSA is 0.7 full-scale and the peak SQNR obtained is $55.5 \mathrm{~dB}$.

\section{BLOCK IMPLEMENTATION}

The next step is to implemented the integrators, the coefficients, the quantizer and the feedback digital to analog converter (DAC). All the circuitry is designed to be implemented in a $65 \mathrm{~nm}$ process. The full CTDSM on circuitry level is shown in Fig. 5. The next subsections describe the topology selection of each block, and how are they realized.

\section{A. Integrators and coefficients}

For the implementation of the integrators an RC-integrator topology is used and it was designed accordingly to [5]

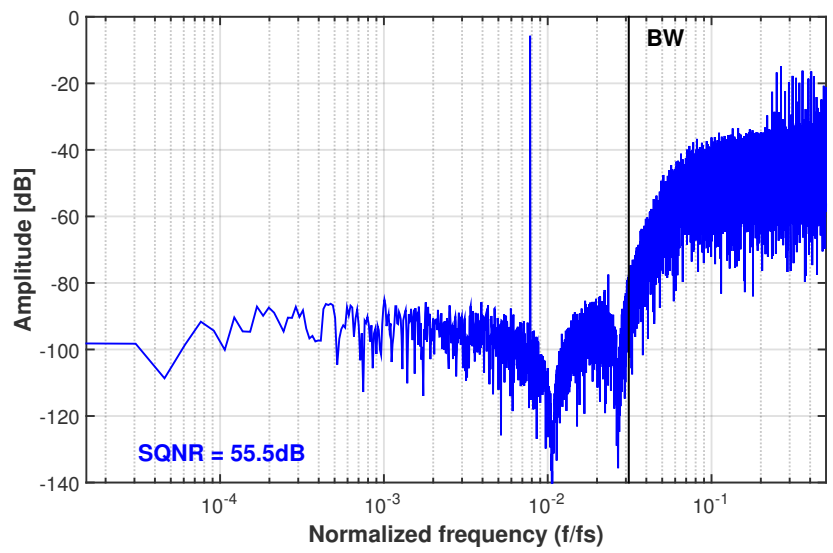

Fig. 4. Frequency spectrum of the continuous-time $\Delta \Sigma$ modulator designed. 


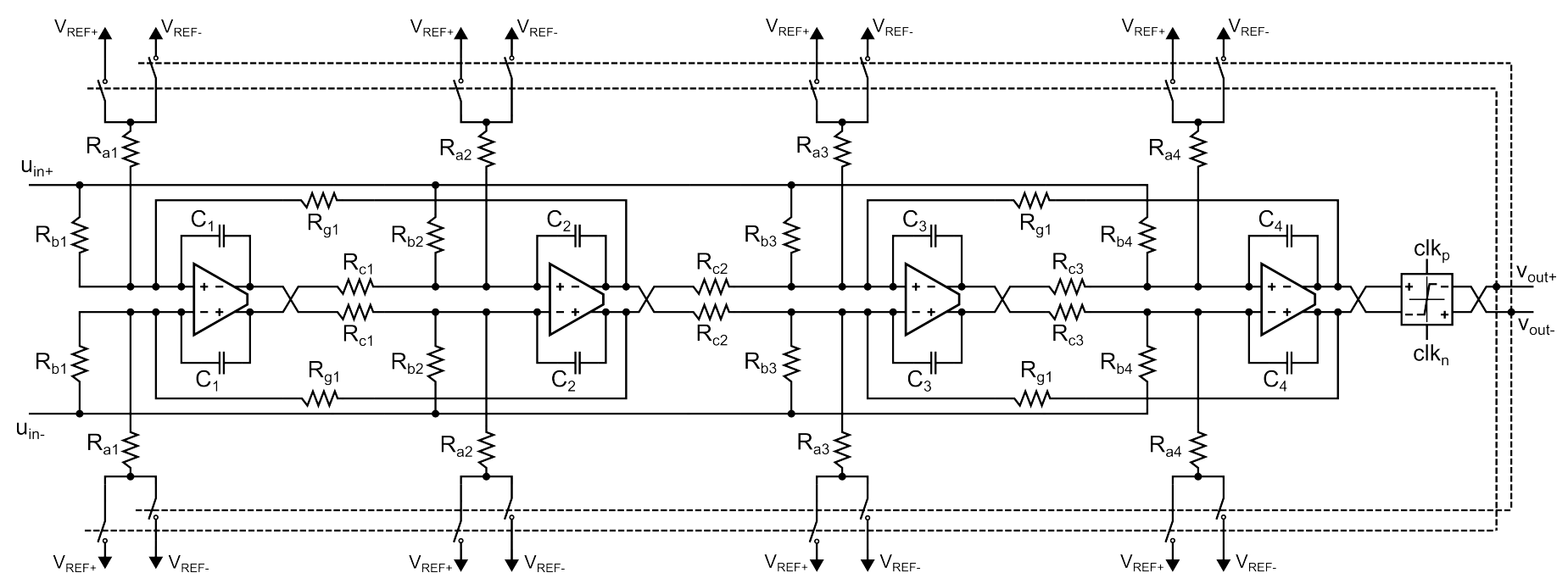

Fig. 5. Continuous-time delta sigma modulator implemented.

It consists of fully-differential operational transconductance amplifier $\left(\mathrm{OTA}_{\mathrm{i}}\right)$, two integrating capacitors $\left(\mathrm{C}_{\mathrm{i}}\right)$ and several resistors which implement the coefficients defined in Section III $\left(a_{i}, b_{i}, c_{i}\right.$ and $\left.g_{i}\right)$. The relationship between the coefficients, $\mathrm{k}_{\mathrm{i}}$, and the value of the resistors $\mathrm{R}_{\mathrm{i}}$ is dictated by (1). The absolute value of the resistors and capacitor is a tradeoff between power consumption and thermal noise which is discussed in Section V-A.

$$
k_{i}=\frac{1}{f_{s} \cdot C_{i} \cdot R_{i}}
$$

This type of integrator was chosen due to its simplicity, its high linearity and high parasitic insensitivity. It was also considered to use gmC integrators since they can provide high frequency operation, but the THD performance of these type of integrators is poor and it is a very critical factor for ultrasound imaging signal quality [4].

\section{B. Quantizer and feedback DAC}

The CTDSM designed has 1-bit output, hence the quantizer can be implemented with a fully-differential comparator. The DACs are realized as voltage feedbacks which consists of a feedback resistor connected to two reference voltages $V_{\text {reft }}$ $\mathrm{V}_{\text {ref- }}$ through two switches controlled by the output of the comparator. This topology was chosen since it is low area demanding, easily controllable and has low parasitics. The feedback pulse shape is chosen to be a non-return to zero due to its less sensitivity to jitter, which is critical at the high operating frequency used, and its low circuitry requirements, which translate into area and power consumption savings.

\section{BLOCK SPECIFICATIONS AND TRADE-OFFS}

Simulations show that the maximum achievable SQNR for this topology is $55.5 \mathrm{~dB}$, however, this number can only be achieved with ideal blocks. The higher the performance of each block the closer the SQNR will be to $55.5 \mathrm{~dB}$. Nonetheless, the circuitry designed is used in portable ultrasound scanners, hence the performance of each block needs to be compromised in favor of reducing the area and power consumption. Furthermore, for a fixed $\mathrm{SNR}_{\text {tot }}$, if the SQNR is lowered the maximum thermal noise allowed needs to be reduced, which also affects the power consumption and area of the circuitry. All these trade-offs between the performance of the blocks, SQNR and thermal noise are difficult to assess due to the complexity of the CTDSM. In order to address these trade-offs, a VerilogA model of the OTA, the comparator and the DACs was created, and a testbench was prepared to simulate the full CTDSM on schematic level. Using this testbench with VerilogA models of the blocks, the designer can easily create trade-off curves by sweeping all the different performance parameters to find a good compromise between block specifications and SQNR.

\section{A. Coefficient capacitor/resistor size}

The coefficients found in Section III impose a relationship between the integrating capacitors and the resistors (1), however, determining the absolute values is a trade-off. The lower the capacitor value, the lower the current to charge it, however the resistors become bigger, hence the thermal noise introduced also increases. The minimum capacitor size of a $65 \mathrm{~nm}$ process is approximately $10 \mathrm{fF}$, which leads to the maximum resistor size of approximately $8 \mathrm{M} \Omega$. The spectral density of the thermal noise generated by such a resistor is $0.36 \mu \mathrm{V} / \sqrt{\mathrm{Hz}}$, which is four orders of magnitude lower compared to the total spectral density of the thermal noise allowed in the circuitry, $3.3 \mathrm{mV} / \sqrt{\mathrm{Hz}}$. Consequently, the thermal noise of the resistors is not a limiting factor, hence the integrating capacitors used should be as small as possible. Capacitor sizes of $100 \mathrm{fF}$ are used for matching purposes and also to make the circuitry more robust to parasitic capacitances.

Another relevant consideration regarding the coefficients is the robustness of the CTDSM to $\mathrm{R}$ and C process variations. In a $65 \mathrm{~nm}$ process, both $\mathrm{R}$ and $\mathrm{C}$ can vary up to $20 \%$. Using the testbench with the VerilogA model of all the blocks, this variation can be introduced in order to see what effect does it have in the CTDSM. The simulations show that by using a 3-bit capacitor trimmeable array for each of the integrator capacitors the SQNR drop due to process variations is less than $0.8 \mathrm{~dB}$. It is important to realize that the OTA needs to be able to handle the maximum capacitance of the trimmeable array, which costs extra current. 
SQNR [dB]
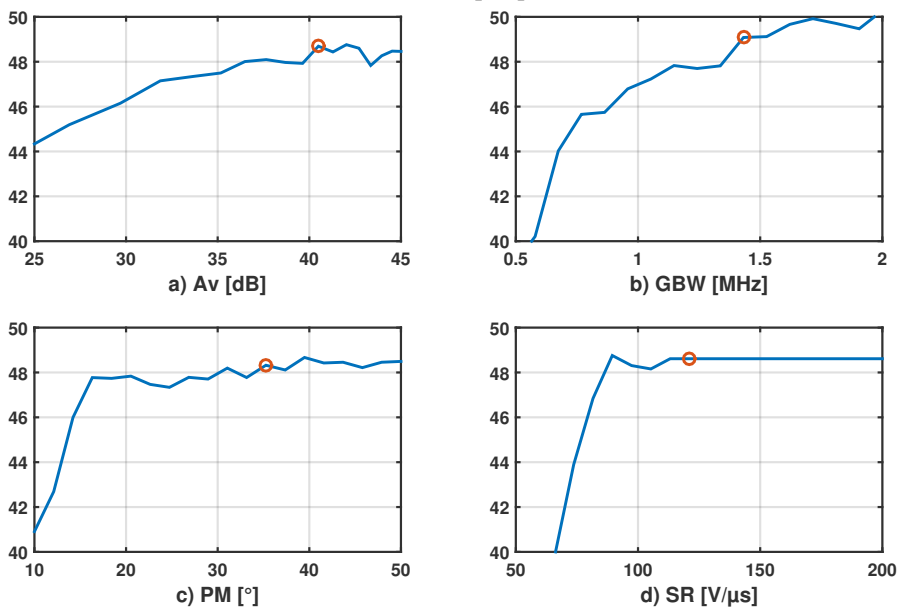

Fig. 6. OTA parameter sweep. SQNR versus: a) $A_{v}$ b) $G B W$ c) PM d) SR.

\section{B. Operational Transconductance Amplifiers}

The OTAs of the CTDSM are the most power consuming parts, hence finding the correct minimum specifications is key to minimize the power consumption of the system. Using the VerilogA model of the fully-differential OTAs, the trade-off curves of the SQNR versus gain $\left(A_{v}\right)$, gain-bandwidth $(\mathrm{GBW})$, phase margin (PM) and slew rate (SR) can be found. The results can be seen in Fig. 6, where an offset of $5 \mathrm{mV}$ is used as a design margin. A good compromise between the OTAs performance parameters and SQNR is found with an $A_{v}$ of $40 \mathrm{~dB}$, a GBW of $1.4 \mathrm{GHz}$, a PM of $35^{\circ}$ and a SR of $120 \mathrm{~V} / \mu \mathrm{s}$. These first OTA specifications lead to a SQNR of $49.2 \mathrm{~dB}$. Readjusting the noise budget to the new SQNR, the maximum spectral density of thermal noise allowed in the circuitry is now $1.74 \mathrm{mV} / \sqrt{\mathrm{Hz}}$. A simple fully-differential OTA with such specifications was quickly designed to assess the approximate magnitude of the thermal noise. Simulations shown a total input referred spectral density of noise of $50 \mu \mathrm{V} / \sqrt{\mathrm{Hz}}$ which is negligible compared to the total thermal noise budget. Consequently, the thermal noise of the OTA is not a design limiting factor. In this design, the same OTA is used in all four integrators for simplicity purposes. However, in future designs the second, third and fourth OTAs can be downscaled lowering the specifications and thereby the power consumption.

\section{Comparator and DACs}

One of the most important factors for the CTDSM stability is the loop delay, which is the time that it takes for the comparator to generate a valid output that can be used as a feedback signal. This loop delay is determined by the speed and transition time of the comparator and DACs. Using the same approach as the OTAs, the VerilogA model of the comparator and DACs are used in order to sweep the total loop delay (ld) and the output transition time (tt). The tradeoff plots are shown in Fig. 7, where an offset of $5 \mathrm{mV}$ is used as a design margin. The specifications for the $1 \mathrm{~d}$ and $\mathrm{tt}$ are set to $0.3 \mathrm{~ns}$ and $55 \mathrm{ps}$ respectively. Similarly to the OTAs the estimated thermal noise generated by the comparator and DACs is negligible compared to the thermal noise budget.

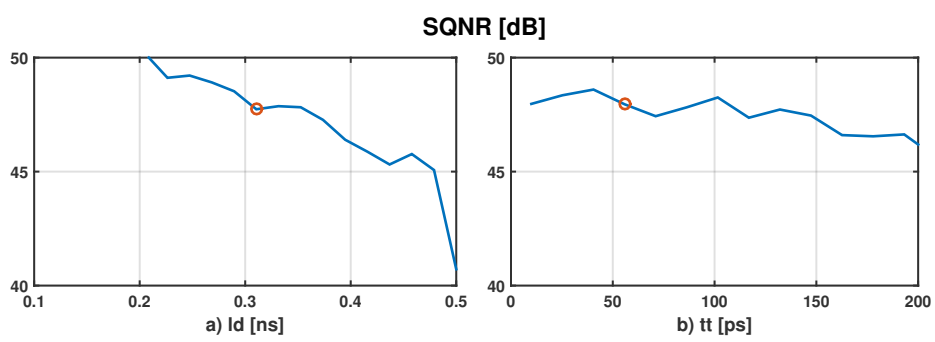

Fig. 7. Comparator and DACs parameter sweep. SQNR versus: a) Loop delay b) Transition time.

\section{DISCUSSION AND FUTURE WORK}

After the trade-off analysis, the values of the resistors and capacitors and also the first specifications for the OTAs, comparator and DACs are defined. The next step is to design the blocks at transistor level using a $65 \mathrm{~nm}$ process. During the design, the performance parameters of the blocks might need to be tweaked due to non-idealities, process corners and mismatch. The design of the OTAs, comparator and DACs are mostly complete and the full $\Delta \Sigma$ modulator design will be sent for fabrication in the next months. The first simulation results show a very high correlation between the results obtained with the VerilogA models and the implemented circuitry, and the expected current consumption of the modulator is $0.9 \mathrm{~mA}$.

\section{CONCLUSIONS}

In this the system level design of a fully-differential continuous-time $\Delta \Sigma$ modulator for portable ultrasound scanners is presented. A fourth order cascade-of-resonators feedback topology with optimal zero placing is used achieving a $\mathrm{SNR}=49.2 \mathrm{~dB}$. The modulator has an OSR of 16, 1bit quantizer and it runs at $\mathrm{a} \mathrm{f}_{\mathrm{s}}$ of $320 \mathrm{MHz}$. The thermal noise of the resistors and OTAs is shown to be negligible due to the low SNR requirements, which inherently leads to a very power efficient implementation. VerilogA models of the OTA, comparator and DACs are used to assess the modulator performance versus the performance parameters of each block generating trade-off curves. The specifications derived for the OTAs are $\mathrm{A}_{\mathrm{v}}=40 \mathrm{~dB}, \mathrm{GBW}=1.4 \mathrm{GHz}, \mathrm{PM}=35^{\circ}$ and $\mathrm{SR}=$ $120 \mathrm{~V} / \mu \mathrm{s}$. The comparator and DACs can allow for a maximum loop delay of $0.3 \mathrm{~ns}$ and a maximum transition time of $55 \mathrm{ps}$.

\section{REFERENCES}

[1] P. Llimós Muntal, D. Ø. Larsen, I. H.H. Jørgensen and E. Bruun, "Integrated reconfigurable high-voltage transmitting circuit for CMUTs" in Analog Integrated Circuits and Signal Processing, Vol. 84, Issue 3, pp.343-352, 2015.

[2] P. Llimós Muntal, D. Ø. Larsen, K. Færch, I. H.H. Jørgensen and E. Bruun, "Integrated Differential High-Voltage Transmitting Circuit for CMUTs" in 13th IEEE International NEW Circuits And Systems, 2015.

[3] R. Schreier and G. C. Temes, Understanding Delta-Sigma Data Converters, Wiley-IEEE Press, 2004.

[4] M. Ortmanns and F. Gerfers, Continuous-Time Sigma-Delta A/D Conversion, Springer, 2006.

[5] N. M.-Villumsen and E. Bruun, "Optimization of Modulator and Circuits for Low Power Continuous-Time Delta-Sigma ADC" in 32nd Norchip Conference, 2014. 